\title{
The Forward Displacement Analysis of 3-RPS Parallel Mechanism Based on Matlab Symbolic Operation \\ Xiao-Dong LIANG ${ }^{1, a}$, Feng-Long YIN ${ }^{1, b}$, Er-Kang LI',c,Hong-Bo LIAO',d
}

${ }^{1}$ Northwest Institute of Nuclear Technology, xi'an 710024,China

axiaodoudong@126.com, byinfenglongwade@163.com,clektt@qq.com,d13975890826@163.com

Keywords: 3-RPS, symbolic operation, position constraint equations, institutions posture figure, direct kinematics.

Abstract. Through analyzing the structural features of the 3-RPS parallel mechanism, establish the position constraint equations of the parallel mechanism, input the initial value, calculate the value of positive solution to location by symbolic operation in the Matlab and get the corresponding institutions posture figure. It shows that the equations for the forward displacement analysis of 3 -RPS can get all the value positive solutions, which is accurate and reliable.

\section{Introduction}

A parallel mechanism is a closed loop mechanism in which the end effector is connected by at least two independent kinematic chains and the basic platform, with two or more two degrees of freedom, and driven in parallel. The mechanism has the advantages of high speed, high strength, high bearing capacity and error characteristics accumulated by domestic and foreign scholars, including Australia Hunt proposed a 3-RPS parallel mechanismto with two dimensional rotation and one dimensional movement, which is a research hotspot ${ }^{[1,2]}$.

\section{Description and establishment of coordinate system for 3-RPS}

The 3-RPS parallel mechanism consists of the lower platform (fixed platform), the upper platform (moving platform) and the 3 identical RPS (revolute pair-moving pair-spherical bearing pair) connecting the upper and lower platforms. With the extension of the moving pair $(\mathrm{P})$, change the position and posture of the upper platform, and realize the two dimensional rotation and one dimensional movement of the upper platform. Establish a fixed coordinate system in $O-X Y Z$ platform in which $O$ is the center of triangle $A_{1} A_{2} A_{3}$ circle, $Y$ axis through the point $A_{1}, Z$ axis perpendicular to the $O X Y$ plane, and the $X$ axis is determined by the right-hand rule, On the basis of the characteristics of institutions. Similarly, on the upper platform to establish dynamic coordinate system $O^{\prime}-X^{\prime} Y^{\prime} Z^{\prime}$, as shown in figure 1 .



Fig.1 3-RPS parallel mechanism 


\section{Forward displacement analysis of 3-RPS}

As shown in Figure 2, the three branched chain vectors are $\vec{W}_{i} \quad(i=1,2,3)$, the angle between the branch and the $O X Y$ plane is $\theta_{i}(i=1,2,3), \quad \phi=30^{\circ}$, the length of the electric cylinder is $L_{i}$ $(i=1,2,3)$, and the length of side of the equilateral triangle in the lower platform is $c$.

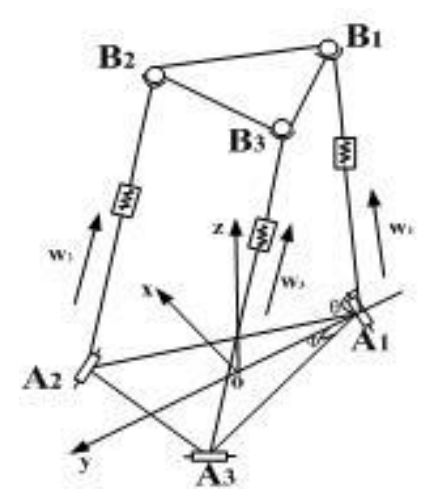

Fig.2 Structure sketch of 3-RPS parallel mechanism

Since the three branches of the mechanism are constrained by the rotational pair, the branch $A_{i} B_{i}(i=1,2,3)$ can only rotate in the plane $O A_{i} B_{i}$. The three branched unit vectors $\vec{W}_{i}$ and the position vectors $\overrightarrow{O A}_{i} \quad(i=1,2,3)$ are as follows.

$$
\begin{aligned}
& \vec{W}_{1}=\cos \theta_{1} \vec{j}+\sin \theta_{1} \vec{k} \\
& \vec{W}_{2}=-\cos \theta_{2} \cos \phi \vec{i}-\cos \theta_{2} \sin \phi \vec{j}+\sin \theta_{2} \vec{k} \\
& \vec{W}_{3}=\cos \theta_{3} \cos \phi \vec{i}-\cos \theta_{3} \sin \phi \vec{j}+\sin \theta_{3} \vec{k}
\end{aligned}
$$

Position vector $\overrightarrow{O A}_{i}(i=1,2,3)$ :

$$
\begin{aligned}
& \overrightarrow{O A}_{1}=-R \vec{j} \\
& \overrightarrow{O A}_{2}=\frac{\sqrt{3}}{2} R \vec{i}+\frac{R}{2} \vec{j} \\
& \overrightarrow{O A}_{3}=-\frac{\sqrt{3}}{2} R \vec{i}+\frac{R}{2} \vec{j}
\end{aligned}
$$

There among, $\vec{i}, \vec{j}$ and $\vec{k}$ are the unit vectors for $X, Y$ and $Z$ axis, $\mathrm{R}$ is the radius of circumcircle in lower platform. According to the laws of vectors $\overrightarrow{O B_{i}}=\overrightarrow{O A_{i}}+L_{i} \vec{W}_{i}$, then

$$
\begin{aligned}
& \left|\overrightarrow{O B}_{1}-\overrightarrow{O B}_{2}\right|^{2}=c^{2} \\
& \left|\overrightarrow{O B}_{2}-\overrightarrow{O B}_{3}\right|^{2}=c^{2}
\end{aligned}
$$


$\left|\overrightarrow{O B}_{3}-\overrightarrow{O B}_{1}\right|^{2}=c^{2}$

Obtained from the above formula that:

$\left\{\begin{array}{l}A_{1} \cos \theta_{1}+B_{1} \cos \theta_{2}+D_{1} \cos \theta_{1} \cos \theta_{2}+E_{1} \sin \theta_{1} \sin \theta_{2}+F_{1}=0 \\ A_{2} \cos \theta_{2}+B_{2} \cos \theta_{3}+D_{2} \cos \theta_{2} \cos \theta_{3}+E_{2} \sin \theta_{2} \sin \theta_{3}+F_{2}=0 \\ A_{3} \cos \theta_{3}+B_{3} \cos \theta_{1}+D_{3} \cos \theta_{1} \cos \theta_{3}+E_{3} \sin \theta_{1} \sin \theta_{3}+F_{3}=0\end{array}\right.$

There among,

$$
\begin{array}{lllll}
A_{1}=-3 R L_{1} & B_{1}=-3 R L_{2} & D_{1}=L_{1} L_{2} & E_{1}=-2 L_{1} L_{2} & F_{1}=3 R^{2}+L_{1}^{2}+L_{2}^{2}-c^{2} \\
A_{2}=-3 R L_{2} & B_{2}=-3 R L_{3} & D_{2}=L_{2} L_{3} & E_{2}=-2 L_{2} L_{3} & F_{2}=3 R^{2}+L_{2}^{2}+L_{3}^{2}-c^{2} \\
A_{3}=-3 R L_{3} & B_{3}=-3 R L_{1} & D_{3}=L_{1} L_{3} & E_{3}=-2 L_{1} L_{3} & F_{3}=3 R^{2}+L_{1}^{2}+L_{3}^{2}-c^{2}
\end{array}
$$

Assign

$x_{i}=\tan \frac{\theta_{i}}{2}$

Then

$$
\sin \theta_{i}=\frac{2 x_{i}}{1+x_{i}{ }^{2}} ; \cos \theta_{i}=\frac{1-x_{i}^{2}}{1+x_{i}^{2}}(i=1,2,3), \text { the Equation (10) can be obtained }
$$

as:

$$
\begin{aligned}
& a_{1} x_{2}^{2}+b_{1} x_{2}+c_{1}=0 \\
& a_{2} x_{3}^{2}+b_{2} x_{3}+c_{2}=0 \\
& a_{3} x_{3}^{2}+b_{3} x_{3}+c_{3}=0
\end{aligned}
$$

There among

$$
\begin{aligned}
& a_{1}=\left(A_{1}-B_{1}-D_{1}+F_{1}\right)+\left(-A_{1}-B_{1}+D_{1}+F_{1}\right) x_{1}^{2} \\
& b_{1}=4 E_{1} x_{1} \\
& c_{1}=\left(A_{1}+B_{1}+D_{1}+F_{1}\right)+\left(-A_{1}+B_{1}-D_{1}+F_{1}\right) x_{1}^{2} \\
& a_{2}=\left(A_{2}-B_{2}-D_{2}+F_{2}\right)+\left(-A_{2}-B_{2}+D_{2}+F_{2}\right) x_{2}^{2} \\
& b_{2}=4 E_{2} x_{2} \\
& c_{2}=\left(A_{2}+B_{2}+D_{2}+F_{2}\right)+\left(-A_{2}+B_{2}-D_{2}+F_{2}\right) x_{2}^{2} \\
& a_{3}=\left(-A_{3}+B_{3}-D_{3}+F_{3}\right)+\left(-A_{3}-B_{3}+D_{3}+F_{3}\right) x_{1}^{2} \\
& b_{3}=4 E_{3} x_{1} \\
& c_{3}=\left(A_{3}+B_{3}+D_{3}+F_{3}\right)+\left(A_{3}-B_{3}-D_{3}+F_{3}\right) x_{1}^{2}
\end{aligned}
$$

Eliminate an $x_{3}$ from formula (13), (14), then:

$\left(a_{2} c_{3}-a_{3} c_{2}\right) x_{3}+\left(b_{2} c_{3}-b_{3} c_{2}\right)=0$ 
Eliminate an $x_{3}^{2}$ from formula (13), (14), then

$$
\left(a_{2} b_{3}-a_{3} b_{2}\right) x_{3}+\left(a_{2} c_{3}-a_{3} c_{2}\right)=0
$$

Eliminate $x_{3}$ from formula (15) and (16), then obtain an algebraic equation with $x_{1}$ and $x_{2}$ which is no higher than about 4 times.

$$
\left(a_{2} c_{3}-a_{3} c_{2}\right)^{2}-\left(a_{2} b_{3}-a_{3} b_{2}\right)\left(b_{2} c_{3}-b_{3} c_{2}\right)=0
$$

Write formula (17) as an explicit expression of $x_{2}$ :

$$
k_{1} x_{2}^{4}+k_{2} x_{2}^{3}+k_{3} x_{2}^{2}+k_{4} x_{2}+k_{5}=0
$$

There among, each coefficient $k_{i}$ is a function of $x_{1}$.

$x_{2}$ multiplies formula (18)

$$
\begin{aligned}
& k_{1} x_{2}^{5}+k_{2} x_{2}^{4}+k_{3} x_{2}^{3}+k_{4} x_{2}^{2}+k_{5} x_{2}=0 \\
& x_{2}, \quad x_{2}^{2}, \quad x_{2}^{3} \text { multiplies formula (18), respectively } \\
& a_{1} x_{2}^{3}+b_{1} x_{2}^{2}+c_{1} x_{2}=0 \\
& a_{1} x_{2}^{4}+b_{1} x_{2}^{3}+c_{1} x_{2}^{2}=0 \\
& a_{1} x_{2}^{5}+b_{1} x_{2}^{4}+c_{1} x_{2}^{3}=0
\end{aligned}
$$

Write the expressions (12), (18), (19), (20), (21) and (22) in matrix form

$$
\left[\begin{array}{cccccc}
0 & k_{1} & k_{2} & k_{3} & k_{4} & k_{5} \\
k_{1} & k_{2} & k_{3} & k_{4} & k_{5} & 0 \\
a_{1} & b_{1} & c_{1} & 0 & 0 & 0 \\
0 & a_{1} & b_{1} & c_{1} & 0 & 0 \\
0 & 0 & a_{1} & b_{1} & c_{1} & 0 \\
0 & 0 & 0 & a_{1} & b_{1} & c_{1}
\end{array}\right]\left[\begin{array}{c}
x_{2}^{5} \\
x_{2}^{4} \\
x_{2}^{3} \\
x_{2}^{2} \\
x_{2} \\
1
\end{array}\right]=0
$$

As $\left[\begin{array}{llllll}x_{2}^{5} & x_{2}^{4} & x_{2}^{3} & x_{2}^{2} & x_{2} & 1\end{array}\right]^{T} \neq 0$, according to the necessary and sufficient conditions, which is the system of homogeneous equations exist nonzero solutions, the following equations can be obtained. 


$$
\left|\begin{array}{cccccc}
0 & k_{1} & k_{2} & k_{3} & k_{4} & k_{5} \\
k_{1} & k_{2} & k_{3} & k_{4} & k_{5} & 0 \\
a_{1} & b_{1} & c_{1} & 0 & 0 & 0 \\
0 & a_{1} & b_{1} & c_{1} & 0 & 0 \\
0 & 0 & a_{1} & b_{1} & c_{1} & 0 \\
0 & 0 & 0 & a_{1} & b_{1} & c_{1}
\end{array}\right|=0
$$

It is a 16 algebraic equation of, the entire solution of $x_{1}$ can be obtained by solving this equation, and the corresponding $x_{2}$ and $x_{3}$ equations can be obtained by formula (23) and formula (16). By substituting the result in formula (11), $\theta_{i}$ can be obtained, and the coordinates of the endpoints of the upper platform in the fixed coordinate system can be calculated with $\overrightarrow{O B_{i}}$.

\section{Numerical example}

Assign the initial values of structure $L_{1}=916 \mathrm{~mm}, L_{2}=958 \mathrm{~mm}, L_{3}=1089 \mathrm{~mm}, \mathrm{R}=800 \mathrm{~mm}$, $c=799 \mathrm{~mm}$, the 16 order equation of $x_{1}$ can be obtained with Matlab symbolic operation.

$$
\begin{aligned}
& 0.2330 \times 10^{5} x_{1}^{16}+0.4085 \times 10^{5} x_{1}^{14}-0.6589 \times 10^{5} x_{1}^{12}+0.1344 \times 10^{5} x_{1}^{10}+0.6512 \times 10^{4} x_{1}^{8} \\
& -0.9611 \times 10^{3} x_{1}^{6}-0.3289 \times 10^{3} x_{1}^{4}-0.1331 \times 10^{2} x_{1}^{2}+0.6987=0
\end{aligned}
$$

The root is

$$
x_{1}=\left\{\begin{array}{cccc} 
\pm 0.1718 & \pm 0.6630 & \pm 0.6907 & \pm 0.7456 \\
\pm 0.3620 i & \pm 0.3860 i & \pm 0.3982 i & \pm 1.6783 i
\end{array}\right\}
$$

The 16 roots can be obtained by solving the 16 order equation. Considering the actual situation, 8 real roots are taken as $x_{1}$ solution, then the corresponding coordinates of endpoint $B_{i}$ in upper platform can be obtained, as shown in table 1.

Table 1 Endpoints $B_{i}$ coordinates on upper platform in mechanism

\begin{tabular}{c|c|c|c}
\hline Class & $\left(x_{B 1}, y_{B 1}, z_{B 1}\right)$ & $\left(x_{B 2}, y_{B 2}, z_{B 2}\right)$ & $\left(x_{B 3}, y_{B 3}, z_{B 3}\right)$ \\
\hline \multirow{3}{*}{1} & $(0,63.92,305.89)$ & $(445.96,257.48,915.01)$ & $(-325.28,187.80,1003.20)$ \\
\cline { 2 - 4 } & $(0,63.92,-305.89)$ & $(445.96,257.48,-915.01)$ & $(-325.28,187.80,-1003.20)$ \\
\hline \multirow{3}{*}{2} & $(0,-443.19,844.17)$ & $(401.34,234.48,901.59)$ & $(-400.18,210.19,1026.10)$ \\
\cline { 2 - 4 } 3 & $(0,-443.19,-844.17)$ & $(401.34,234.48,-901.59)$ & $(-400.18,210.19,-1026.10)$ \\
\cline { 2 - 4 } 3 & $(0,-475.52,857.12)$ & $(371.75,214.63,883.77)$ & $(232.16,-134.04,214.06)$ \\
\cline { 2 - 4 } & $(0,-475.52,-857.12)$ & $(371.75,214.63,-883.77)$ & $(232.16,-134.04,-214.06)$ \\
\hline \multirow{3}{*}{4} & $(0,-538.46,878.37)$ & $(-102.98,-59.46,272.22)$ & $(-299.04,172.65,989.88)$ \\
\cline { 2 - 4 } & $(0,-538.46,-878.37)$ & $(-102.98,-59.46,-272.22)$ & $(-299.04,172.65,-989.88)$ \\
\hline
\end{tabular}

According to the platform endpoint coordinates shown in Table 1, the position of the upper platform is plotted, as shown in Figure 3. 


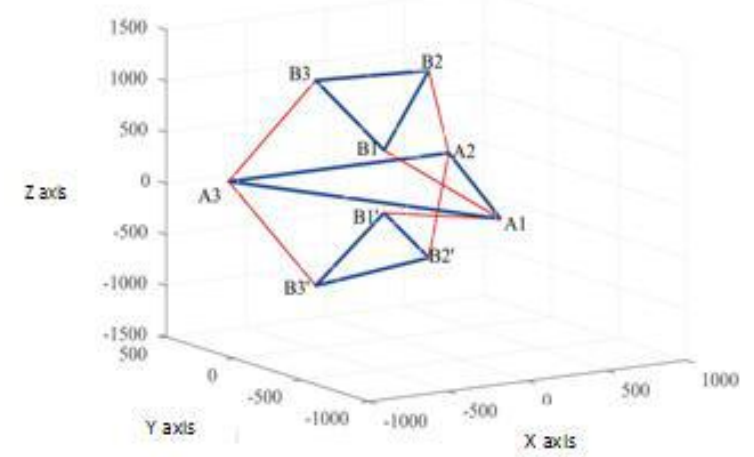

(1)



(3)

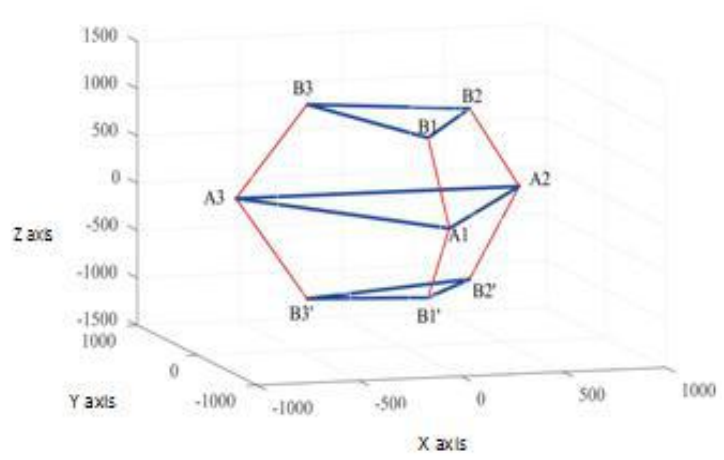

(2)

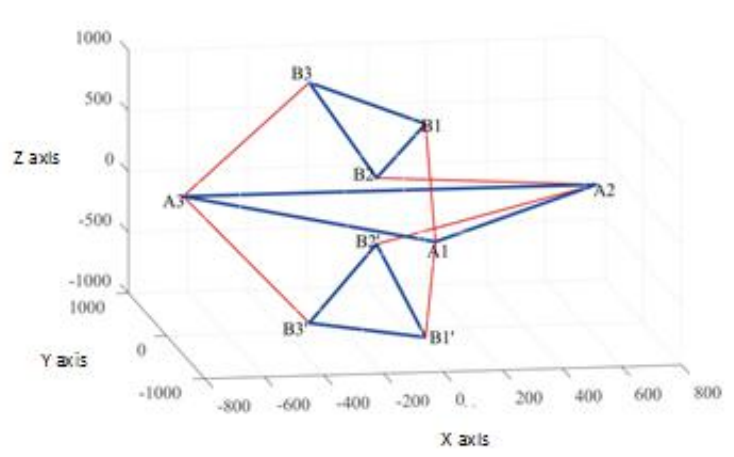

(4)

Fig. 3 position and posture of platform on platform

\section{Conclusion}

In this article, according to the geometrical relations of 3-RPS parallel mechanism, establish the equation of position constraint with analytic method. Work out the 16 sets of solutions for position constraint equations with Matlab symbol operation, and then obtain the corresponding mechanism posture figures. The results show that the method is accurate and reliable.

\section{Reference}

[1] MEI Jiang-ping, GAO Ben, TAN Yang, etc. Kinematic analysis of 3-SPR parallel mechanism [J]. Proceedings of the agricultural mechanical society, 2012, 43 (8): 215-220.

[2] ZHENG Kui-jing, CUI Pei, GUO Hai-jun. Kinematic and static characteristics analysis of 3-RPS parallel mechanism [J]. Mechanical design, 2011, 28 (9): 28-33.

[3] HUANG Zhen, ZHAO Yong-sheng, ZHAO Tie-shi. Advanced space science [M]. Beijing: Higher Education Press, 2006.

[4] LI Bin, HUANG Tian, LIU Hai-tao, etc. Module position analysis of three DOF parallel mechanism of Exechon hybrid robot [J]. Chinese mechanical engineering, 2010, 21 (23): 2785-2789.

[5] WANG Hai-ong, YANG Bing-heng, BI Yu-quan, etc. Kinematic analysis of a new type of parallel mechanism with three DOF [J]. China manufacturing information, 2011, 40 (1): 29-32.

[6] FAN Li-hua. Research on kinematics and dynamics of three DOF parallel kinematic machine [D]. Chengdu: Xihua University, 2005.

[7] LIU Lin-shan. The design method study of 3-PRS parallel mechanism [D] Tianjin: Tianjin University, 2007.

[8]Meng-Shiun Tsai,Ting-Nung Shiau. Direct Kinematic Analysis of a 3-PRS Parallel Mechanism [J]. Mechanism and Machine Theory 38 (2003) 71-83.

[9] Jinwook Kim,F.C.Park. Direct kinematic analysis of 3-RS parallel mechanism[J]. Mechanism and Machine Theory 36 (2001) 1121-1134. 